CAR-TR-701

CCR-89-08901

CS-TR-3219

JSA $91-5$

February 1994

\title{
Minimum Enclosures with Specified Angles
}

\author{
David M. Mount \\ Department of Computer Science and \\ Institute for Advanced Computer Studies \\ University of Maryland \\ College Park, MD 20742
}

\author{
Ruth Silverman \\ Department of Computer Science \\ University of the District of Columbia \\ Washington, DC 20008 \\ and \\ Center for Automation Research \\ University of Maryland \\ College Park, MD 20742
}

\begin{abstract}
Given a convex polygon $P$, an $m$-envelope is a convex $m$-sided polygon that contains $P$. Given any convex polygon $P$, and any sequence of $m \geq 3$ angles $A=\left\langle\alpha_{1}, \alpha_{2}, \ldots, \alpha_{m}\right\rangle$, we consider the problem of computing the minimum area $m$-envelope for $P$ whose counterclockwise sequence of exterior angles is given by $A$. We show that such envelopes can be computed in $O(n m \log m)$ time. The main result on which the correctness of the algorithm rests is a flushness condition stating that for any locally minimum enclosure with specified angles, one of its sides must be collinear with one of the sides of $P$.
\end{abstract}

The support of the National Science Foundation under Grant CCR-89-08901, the Bureau of the Census under grant JSA 91-5, and the University of the District of Columbia under a Faculty Senate Summer Research Grant is gratefully acknowledged, as is the help of Sandy German in preparing this paper. A preliminary version of this paper appeared in Vision Geometry, R.A. Melter and A.Y. Wu, Editors, Proc. SPIE 1832, 1993, 80-91. 


\section{Introduction}

Given a convex polygon $P$, for $m \geq 3$ we define an $m$-envelope for $P$ to be an $m$-sided convex polygon that encloses $P$. In this paper we consider the problem of computing the minimum area $m$-envelope where the angles of the envelope are specified. More formally the problem is, given a planar convex $n$-gon $P$, and a sequence of $m$ prescribed angles $A=\left\langle\alpha_{1}, \alpha_{2}, \ldots, \alpha_{m}\right\rangle$, determine a minimum area $m$-envelope having these prescribed exterior angles in counterclockwise sequence about its boundary. Clearly this sequence of angles must satisfy

$$
m \geq 3 \quad \text { and } \quad 0<\alpha_{i}<\pi \quad \text { and } \quad \sum_{i=1}^{n} \alpha_{i}=2 \pi .
$$

Although the exterior angles are fixed, the lengths of the edges are not specified. We allow for the degenerate case of envelope edges of length zero, implying that the enclosure may have fewer than $m$ sides.

Our main result is an $O(n m \log m)$ time and $O(n+m)$ space algorithm for this problem. This is $O(n)$ time and space if $m$ is a constant. The algorithm itself is a straightforward application of the method of rotating calipers [16], but some care is needed to efficiently maintain the area of envelopes. Perhaps the most interesting aspect of our result is a proof that any locally minimum $m$-envelope with given exterior angles must be flush with an edge of $P$, meaning that one of the edges of the enclosure must be collinear with one of the edges of $P$. Flushness is a common finiteness condition used to restrict the otherwise infinite search performed in many geometric optimization algorithms. For many existing rotating calipers solutions, establishing flushness is a relatively easy matter (but there are exceptions

[5]). However, for this problem the proof of the flushness condition is much subtler because any rotation of a single side of the $m$-envelope affects all $m$ sides (because of the angle constraints). We prove the flushness condition by an induction argument that reduces the problem to the case of a triangle with angle constraints.

Computing minimal convex enclosures for geometric objects is a fundamental geometric problem. Because the enclosures are assumed to be convex, it is sufficient to consider computing an enclosure for the convex hull of the geometric object in question. This problem arises in applications in which one wishes to find an approximation to a convex object that 
is simpler in the sense of having a smaller number of sides. The problem of computing minimum convex polygonal enclosures of $m$ sides for an $n$-sided convex polygon (without angle constraints) was considered originally by Boyce, Dobkin, Drysdale, and Guibas [2] and later by Aggarwal and Park [1], who showed that the problem can be solved in $O(n m)$ time.

It is often of practical value to impose additional shape constraints on the enclosure, for example, the constraint that a four-sided enclosure be a rectangle, or that a three-sided enclosure be an equilateral triangle. Observe that both of these examples can be cast in terms of computing enclosures with given angle constraints. Work of this type includes that of Toussaint [16], who gave a linear time algorithm for finding the smallest enclosing rectangle; Depano and Aggarwal [6], who gave linear time algorithms for computing the smallest enclosing equilateral triangle and square; and $\mathrm{O}^{\prime}$ Rourke [13], who presented an $O\left(n^{3}\right)$ algorithm for the three-dimensional problem of computing the smallest rectangular box enclosing a convex polytope.

Our algorithm, like many of those listed above, is based on the method of "rotating calipers." This method has been used, for example, for the problem of determining the smallest triangle enclosing a given convex polygon, by Klee and Laskowski [9] and O’Rourke, et al. [14]. It has also been used by Dobkin and Snyder [7] for the problem of computing the largest triangle enclosed in a convex polygon (see also Chandran and Mount [3]). Other examples include algorithms for packing and covering the plane with convex polygons (see Mount and Silverman [12] and Mount [11]).

\section{Background}

We begin with some observations about the nature of locally minimal enclosures with specified exterior angles for a given convex polygon $P$. Each edge of any such minimal enclosure must contact the boundary of $P$, for if not, we could decrease the area of any convex enclosure having a nontouching edge by translating such an edge parallel to itself inwards towards $P^{\prime}$ s boundary. It follows as a consequence that degenerate edges of zero length must be allowed, or else minimum enclosures do not exist. Suppose for example that $P$ is a square and we seek a minimum 5 -sided enclosure with the exterior angle sequence $\langle\pi / 2, \pi / 2, \pi / 2, \pi / 4, \pi / 4\rangle$. 
It is easy to see that for every $\epsilon>0$ there is an enclosure with these exterior angles whose area differs from $P$ by $\epsilon$ (see Fig. 1(a)). The limiting enclosure is equal to $P$ and has one side of length zero, that is, two concurrent vertices.

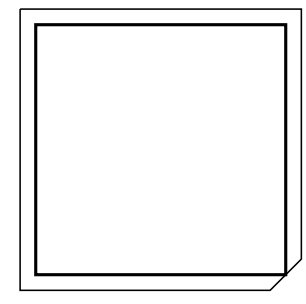

(a)

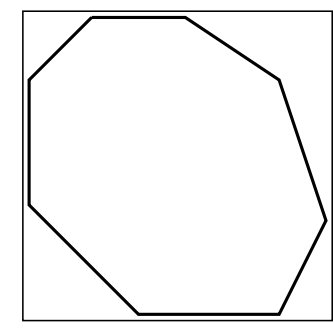

(b)

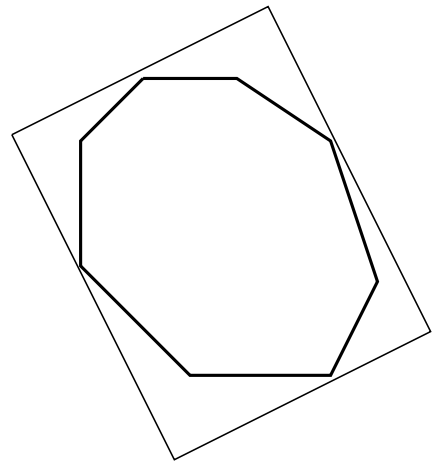

(c)

Figure 1: Fixed angle enclosures.

Henceforth, assume that the edges of any polygon are directed counterclockwise around the boundary of the polygon. Define the angle of a directed line to be the angle formed between the unit vector on the $x$-axis and a unit vector directed parallel to the line. For each angle $\theta$, there is a unique directed line supporting $P$ at this angle, such that $P$ lies to the left of the supporting line. Given a sequence of exterior angles $A=\left\langle\alpha_{1}, \ldots, \alpha_{m}\right\rangle$ it will be notationally convenient to define the following sequence of angle offsets by computing prefix sums of the $\alpha$ 's. Let $B=\left\langle\beta_{1}, \ldots, \beta_{m}\right\rangle$ where

$$
\beta_{i}=\sum_{1 \leq j<i} \alpha_{i}
$$

(and $\beta_{1}=0$ ). For a given angle $\theta$, there is a unique $m$-envelope with sides contacting $P$, and satisfying the exterior angle constraints, formed by taking the intersection of the left side halfspaces of the $m$ supporting lines for $P$ at the angles

$$
\beta_{1}+\theta, \beta_{2}+\theta, \ldots, \beta_{m}+\theta .
$$

Let $\operatorname{Env}(\theta)$ denote this $m$-envelope. In Fig. 1(b) and (c) we show $\operatorname{Env}(\theta)$ for two different values of $\theta$. From our earlier observation that the edges of any minimum envelope contact the polygon, it follows that it suffices to compute

$$
\inf _{0 \leq \theta<2 \pi} \operatorname{Area}(\operatorname{Env}(\theta)) .
$$


As $\theta$ varies continuously from 0 to $2 \pi$, define the contact points of $\operatorname{Env}(\theta)$ to be the sequence of vertices of $P$ that the sides of $\operatorname{Env}(\theta)$ contacts. If an edge of $\operatorname{Env}(\theta)$ is flush with an edge of $P$ then we take the contact point to be the counterclockwise endpoint of this edge of $P$. An angle is critical if such a flush contact occurs. Equivalently, an angle $\theta$ is critical if and only if, for some counterclockwise directed edge $e$ of $P$ and for some $i, 1 \leq i \leq m$, $\beta_{i}+\theta$ is equal to the orientation of $e$. As $\theta$ ranges from 0 to $2 \pi$ each of the $m$ sides of the envelope will become flush exactly once with each of the $n$ sides of $P$, and hence there are at most $n \cdot m$ distinct critical angles (fewer if multiple sides are simultaneously flush). The main result establishing the correctness of our algorithm is the following flushness condition.

Theorem 2.1 (Flushness Condition)

$\operatorname{Env}(\theta)$ is locally minimum with respect to area only if $\theta$ is a critical angle, that is, if an edge of $\operatorname{Env}(\theta)$ is flush with an edge of the polygon.

This theorem is proved in Section 4. For now, assuming this result we can outline the algorithm. As mentioned earlier, the algorithm operates by the method of rotating calipers. We begin by computing the initial envelope, $\operatorname{Env}(0)$, by determining the contact points of the directed supporting lines for each angle in $B$. Since the angles of this sequence are sorted, we can do this in $O(n+m)$ time by a single counterclockwise scan around the boundary of $A$. Once the contact points are known, it is an easy matter to compute the area of the resulting envelope in $O(m)$ time. (We assume a real-RAM model of computation in which arithmetic operations, comparisons, square roots, and trigonometric functions can be performed in constant time [15].)

The algorithm operates in stages, each one advancing from one critical angle to the next. To determine the next critical event, for each edge of the envelope we compute the next angle $\theta$ at which it becomes flush with the edge of $P$ lying just counterclockwise of the contact point. Among these $m$ angles we select the smallest to be the next critical angle. (In general there may be ties for the next critical angle, but it will simplify the presentation to assume that they are distinct.) By storing these angles and the associated contact points and envelope sides in a priority queue [4] we can extract the minimum angle in $O(\log m)$ time. Given the next critical angle we can update the contact point that has changed in constant 
time (by sliding it to the next vertex in counterclockwise order) and insert an event angle for the next edge into the priority queue. This is repeated $O(\mathrm{~nm})$ times until the critical angle cycles through a full rotation. The total time spent accessing the priority queue is $O(n m \log m)$, and the space needed for the priority queue and polygon together is $O(n+m)$. At each critical angle we must update the current area for the next envelope. We consider how this is done later. When the algorithm is completed, then the smallest polygon found so far is reported. We summarize the algorithm below. It is given the polygon $P$ and the sequence $\alpha$ of exterior angles.

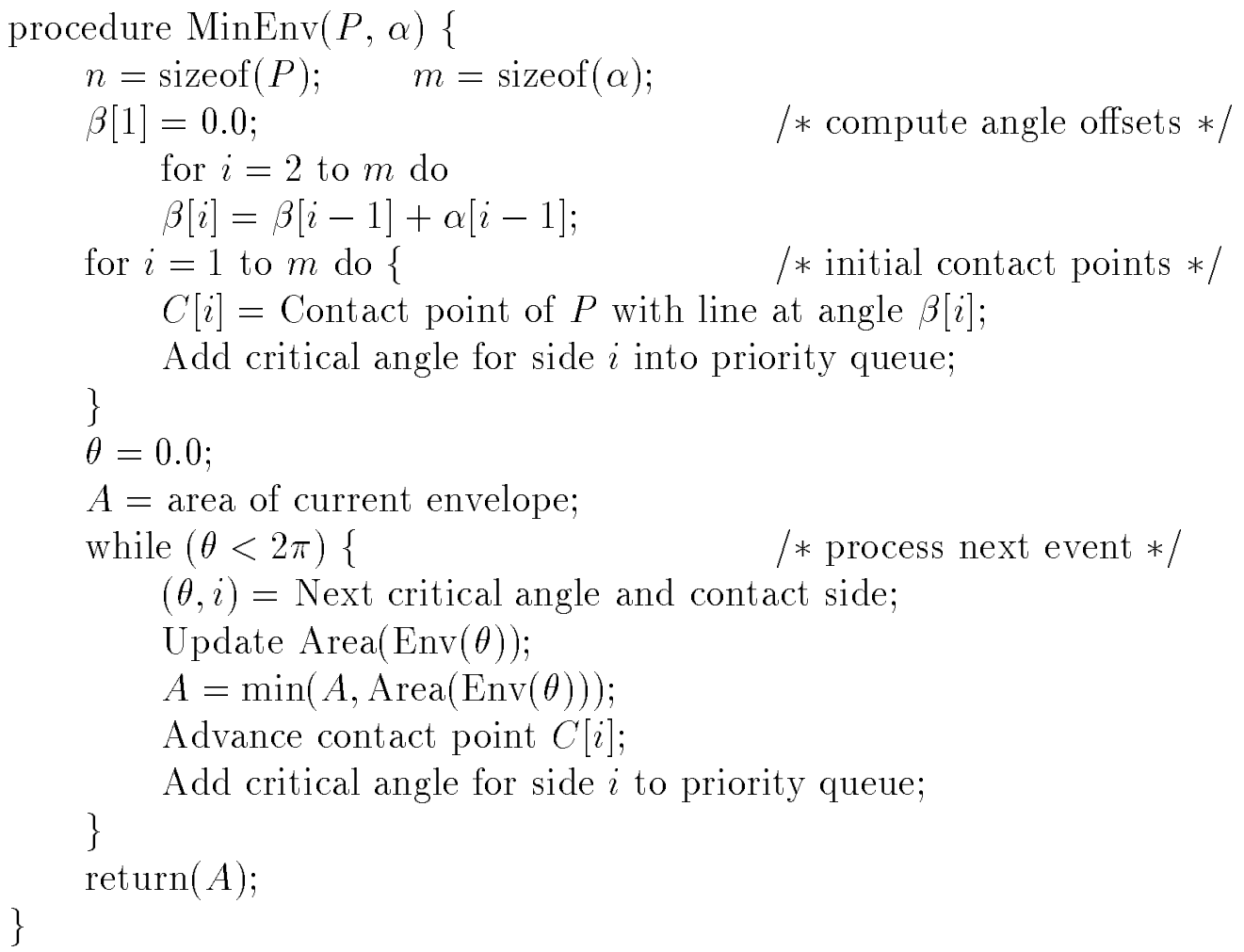

There are two missing elements needed to complete the description of the algorithm and establish its correctness. The first is how the area can be updated efficiently from one critical placement to the next. This must be done in $O(\log m)$ time to achieve the desired running time. We show how this is done in the next section. The second element, as mentioned above, is the proof of Theorem 2.1, which shows that it suffices to consider envelopes only at critical angles. This is proved in Section 4. 


\section{Area Computation}

In this section we describe how the area of the $m$-envelope, $\operatorname{Env}(\theta)$, is updated as we move from one critical angle to the next. Intuitively, between critical angles the contact points with the polygon $P$ remain unchanged, and hence the area varies with $\theta$ in a smooth continuous manner. We will see that this area function can be described by a simple formula, which is a function of $\theta$, whose coefficients are functions of the sequence of exterior angles $A$ (or the derived sequence $B$ of offset angles) and the position of these contact points. Of course the length of such a formula (that is, the number of symbols in the formula) would expected to grow at least proportionally with $m$, implying that its evaluation would require $O(m)$ time, and not the $O(\log m)$ time that we desire. However, we show how to express the formula in terms of a tree-structured collection of $O(m)$ formulas each of constant complexity, such that when we arrive at a critical angle, this collection can be updated in $O(\log m)$ time.

We begin by deriving the formulas for the area at a local level. Let $c_{1}, c_{2}, \ldots, c_{m}$ denote the contact points for the current envelope, $\operatorname{Env}(\theta)$, where $c_{i}$ is the contact with the edge at angle $\beta_{i}$. Let $a_{i}(\theta)$ denote the vertex of $\operatorname{Env}(\theta)$ that lies between points $c_{i}$ and $c_{i+1}$. The set of contact points changes discretely only at critical angles (depending on which edge becomes flush). Let $H$ denote the convex hull of these contact points, and for $1 \leq i \leq m$, let $T_{i}(\theta)$ denote the triangle whose vertices are $c_{i}, c_{i+1}$, and $a_{i}$, see Fig. 2(a). (Throughout, indexing is performed cyclically modulo $m$ so that $c_{m+1}=c_{1}$.) If the envelope contains edges of length zero, then $T_{i}(\theta)$ may degenerate to a single point. Observe that $\operatorname{Env}(\theta)$ is equal to the disjoint union of $H$ and the $m$ triangles $T_{i}(\theta)$. And hence the area of $\operatorname{Env}(\theta)$ can be computed by summing the area of $H$ and the areas of these triangles.

As $\theta$ varies between two critical angles, $H$ does not change, and hence contributes a constant additive term to the area of $\operatorname{Env}(\theta)$, whereas the area of $T_{i}(\theta)$ varies continuously. Let us explore this variation between critical angles. First observe that because the angle at vertex $a_{i}(\theta)$ is fixed at $\pi-\alpha_{i}$ and the contact points are fixed between critical angles, as $\theta$ varies the vertex $a_{i}(\theta)$ travels along an arc of a circle passing through $c_{i}$ and $c_{i+1}$ of arc size $2 \alpha_{i}$. As far as the algorithm is concerned it is not possible for $a_{i}(\theta)$ to travel along the entire boundary of this circle, but it will be convenient for us to extend the definition of $T_{i}(\theta)$ for 


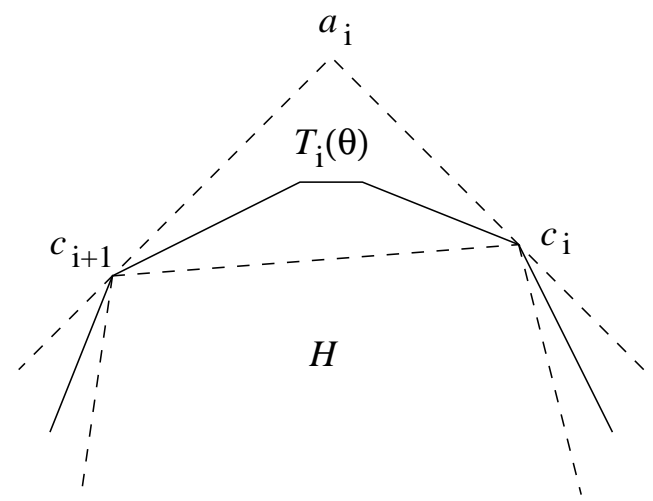

(a)

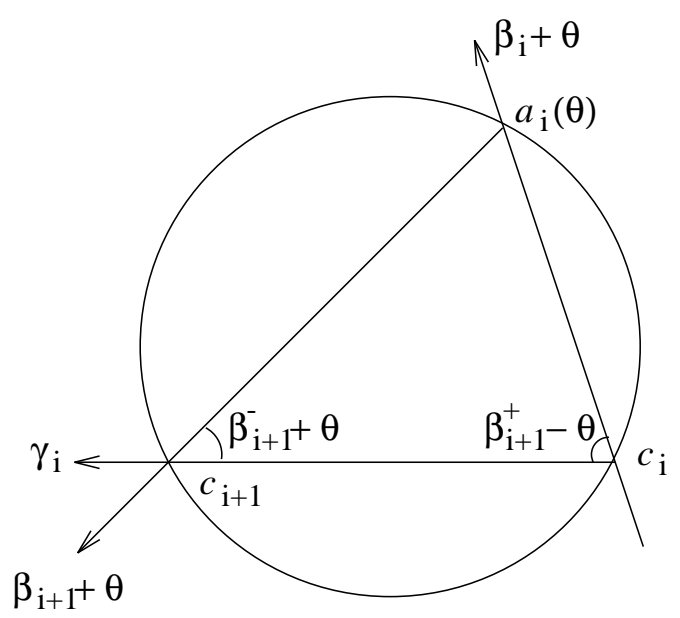

(b)

Figure 2: Local area computation.

the entire range of $\theta$ from 0 to $2 \pi$ by defining $a_{i}(\theta)$ to be the intersection point of a line passing through $c_{i}$ at angle $\beta_{i}+\theta$ and a line passing through $c_{i+1}$ at angle $\beta_{i+1}+\theta$. It will also be convenient to make the convention that the "area" of $T_{i}(\theta)$, denoted $\operatorname{Area}_{i}(\theta)$, is a signed quantity that is negative as $a_{i}$ passes to the other side of the chord $\overline{c_{i} c_{i+1}}$. We call the resulting figure an extended envelope.

Let $\gamma_{i}$ denote the angle of the directed segment from $c_{i}$ to $c_{i+1}$. It will be convenient notationally to define

$$
\beta_{i}^{+}=\gamma_{i}-\beta_{i} \quad \text { and } \quad \beta_{i}^{-}=\beta_{i}-\gamma_{i-1} \quad \text { and } \quad \beta_{i}^{ \pm}=\beta_{i}^{+}-\beta_{i+1}^{-} .
$$

Now referring to Fig. 2(b), the side of $\operatorname{Env}(\theta)$ contacting $c_{i}$ has the angle $\beta_{i}+\theta$, and hence forms the angle

$$
\gamma_{i}-\left(\beta_{i}+\theta\right)=\beta_{i}^{+}-\theta
$$

with chord $\overline{c_{i} c_{i+1}}$. The side of $\operatorname{Env}(\theta)$ contacting $c_{i+1}$ has the angle $\beta_{i+1}+\theta$, and forms the angle

$$
\left(\beta_{i+1}+\theta\right)-\gamma_{i}=\beta_{i+1}^{-}+\theta
$$

with the same chord. Letting $d_{i}$ denote the length of the chord $\overline{c_{i} c_{i+1}}$ we apply a standard formula for the area of a triangle [8] (which is an immediate consequence of the included 
angle formula for the area of a triangle and the law of sines), yielding

$$
\operatorname{Area}_{i}(\theta)=\frac{d_{i}^{2} \sin \left(\beta_{i}^{+}-\theta\right) \sin \left(\beta_{i+1}^{-}+\theta\right)}{2 \sin \alpha_{i}} .
$$

It is a straightforward matter to verify that this signed area function satisfies the convention mentioned earlier as $\theta$ varies from 0 to $2 \pi$.

For now let us drop the subscripts to simplify the notation. Expanding the above formula we have

$$
\operatorname{Area}(\theta)=\frac{d^{2}}{2 \sin \alpha} \sin \left(\beta^{+}-\theta\right) \sin \left(\beta^{-}+\theta\right),
$$

which, after tediously expanding, multiplying, combining terms, and then further simplifying by expressing in terms of $2 \theta$ and $\beta^{ \pm}$, leads to

$$
\operatorname{Area}_{i}(\theta)=\frac{d_{i}^{2}}{4 \sin \alpha_{i}}\left(\sin \beta_{i}^{ \pm} \sin 2 \theta+\cos \beta_{i}^{ \pm} \cos 2 \theta-\cos \left(\beta_{i}^{+}+\beta_{i+1}^{-}\right)\right) \text {. }
$$

Given that $\beta_{i}^{+}+\beta_{i+1}^{-}=\beta_{i+1}-\beta_{i}=\alpha_{i}$, we can formulate the area function for each triangle in either of the following simple forms.

LEMMA 3.1 Consider the sequence of contact points $\left\langle c_{1}, c_{2}, \ldots, c_{m}\right\rangle$ arising in the computation of the envelope, let $\operatorname{Area}_{i}(\theta)$ denote the area of the triangle $T_{i}(\theta)$ bounded between the contact points $c_{i}$ and $c_{i+1}$, and let Area $(\theta)$ denote the total area function.

(i) $\operatorname{Area}_{i}(\theta)=\left(d_{i}^{2} /\left(4 \sin \alpha_{i}\right)\right)\left(\sin \beta_{i}^{ \pm} \sin 2 \theta+\cos \beta_{i}^{ \pm} \cos 2 \theta-\cos \alpha_{i}\right)$.

(ii) $\operatorname{Area}_{i}(\theta)=\left(d_{i}^{2} /\left(4 \sin \alpha_{i}\right)\right)\left(\cos \left(2 \theta-\beta_{i}^{ \pm}\right)-\cos \alpha_{i}\right)$.

(iii) Area $(\theta)$ is of the general form $a(\cos (2 \theta+b))+c$, for constants $a, b, c$ independent of $\theta$.

PROOF: Parts (i) and (ii) follow from the derivation given above and standard trigonometric identities, and part (iii) follows from the facts that (1) each function $\operatorname{Area}_{i}(\theta)$ is of the form $a(\cos (2 \theta+b))+c,(2)$ it is well known from trigonometry that the sum of two functions of this form is of this same form, and $(3) \operatorname{Area}(\theta)=\operatorname{Area}(H)+\sum_{i} \operatorname{Area}_{i}(\theta)$.

Part (i) of this lemma describes Area $_{i}$ as a linear function of $\sin 2 \theta$ and $\cos 2 \theta$ of the form $p_{i} \sin 2 \theta+q_{i} \cos 2 \theta+r_{i}$, where the triple of coefficients, $\left(p_{i}, q_{i}, r_{i}\right)$, depends only on the 
local structure of $T_{i}$. Henceforth we assume that each triangle's area function is represented symbolically as such a triple. Let $\operatorname{Area}_{j, k}(\theta)$ denote the sum of the areas of consecutive triangles from $T_{j}(\theta)$ through $T_{k}(\theta)$, that is,

$$
\operatorname{Area}_{j, k}(\theta)=\sum_{j \leq i \leq k} T_{i}(\theta)
$$

As before, although the function Area $_{j, k}$ is meaningful to the algorithm only between a pair of critical angles, we will extend this function over the entire period $0 \leq \theta<2 \pi$. This function can be represented by simply storing the corresponding sums of coefficients

$$
p_{j, k}=\sum_{j \leq i \leq k} p_{i} \quad \text { and } \quad q_{j, k}=\sum_{j \leq i \leq k} q_{i} \quad \text { and } \quad r_{j, k}=\sum_{j \leq i \leq k} r_{i}
$$

We can now discuss how area calculations are performed. We use a standard technique of representing the function as the sum of functions stored in a binary tree. We construct a balanced binary tree of height $O(\log m)$ whose leaves, from left to right, contain the vector representations $\left(p_{i}, q_{i}, r_{i}\right)$ of Area $_{i}$ for $1 \leq i \leq m$. Each internal node is the root of a subtree whose leaves are associated with a consecutive set of triangles $T_{i}$, say for $j \leq i \leq k$. At this node we store the vector coefficients $\left(p_{j, k}, q_{j, k}, r_{j, k}\right)$ representing the aggregate area function Area $_{j, k}$.

Initializing the leaves of this tree at the start of the algorithm can be performed in $O(m)$ time, given the initial set of contact points. We can compute the coefficients at the internal nodes of the tree by simply summing the vectors of coefficients of the two children. At the same time we also compute the area of $H$, the convex hull of the contact points, in $O(m)$ time.

As the algorithm is running, the area of $\operatorname{Env}(\theta)$ can be computed in constant time by computing the area function at the root of the tree, and adding to this the area of the convex hull of the contact points, which is maintained separately. When a contact point $c_{i}$ changes locally, implying a change in the adjoining triangles $T_{i}$ and $T_{i-1}$, we perform the following steps.

- Update the area of the new convex hull of the contact points. Since only one point $c_{i}$ has changed position, and it lies in the same relative position within the convex hull, 
this can be done by subtracting the area of the old triangle formed by $c_{i-1}, c_{i}$ and $c_{i+1}$ and adding in the area of the new triangle after modifying $c_{i}$.

- Compute the updated area functions for $T_{i}$ and $T_{i-1}$. Each can be done in constant time.

- For each ancestor of $T_{i}$ or $T_{i-1}$, recompute its area function. Since the height of the tree is $O(\log m)$, this can be done in $O(\log m)$ time.

In summary, we can maintain a data structure which allows us to compute the area of $\operatorname{Env}(\theta)$ in constant time for any $\theta$, and can be initialized in $O(n)$ time and updated in $O(\log m)$ time. As mentioned earlier, the total number of updates is $O(n m)$, leading to a total cost of $O(n m \log m)$.

\section{Flushness Condition}

Recall from the introduction that an angle $\theta$ is critical if one of the edges of the envelope, $\operatorname{Env}(\theta)$ is collinear, that is flush, with an edge of the polygon $P$. In this section we prove Theorem 2.1, which states that the minimum area $m$-envelope with specified exterior angles will be equal to $\operatorname{Env}(\theta)$ for some critical angle $\theta$, thus reducing the search to a set of angles of size $O(n m)$.

Consider the sequence $B$ of $m$ offset angles $\left\langle\beta_{1}, \beta_{2}, \ldots, \beta_{m}\right\rangle$ introduced earlier. As before, we consider the current set of $m$ contact points of the envelope, $\left\{c_{1}, c_{2}, \ldots, c_{m}\right\}$, and describe the area of the envelope as a function of $\theta, \operatorname{Area}(\theta)$. As before we are only interested in the value of this function between two critical angles, but we extend its definition over the period $2 \pi$, by considering the extended envelope with these contact points. Because this function represents the area of $\operatorname{Env}(\theta)$ in the interval between two critical angles, to prove that local minima occur only at critical angles it suffices to show that this function has no local minima in the interval between critical angles. This function is positive between two critical angles, but may be nonpositive when extended to the full $2 \pi$ range of angles. Thus it suffices to show that any local minima occur when the function value is nonpositive, since any such 
angles cannot lie in the interval between critical angles. The rest of this section is devoted to establishing this.

We prove this by an inductive argument on $m$, the number of sides in the envelope. By our hypothesis that no exterior angle exceeds $\pi$, it follows that if we consider $m$ unit vectors given the same orientations as the offset angles $B$, these vectors positively span the plane (for otherwise there would be no envelope of finite area having sides at these angles). If $m \geq 4$ then there exists a subset of angles of size $m-1$ that positively spans the plane. By successively removing one angle at a time we can form a sequence of area functions for envelopes of $m, m-1, \ldots, 3$ sides

$$
\text { Area }^{(m)}(\theta), \text { Area }^{(m-1)}(\theta), \ldots, \text { Area }^{(3)}(\theta)
$$

See Fig. 3.

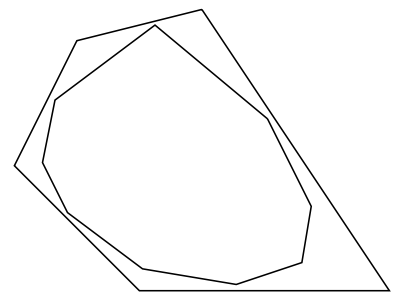

$\operatorname{Area}^{(5)}(\theta)$

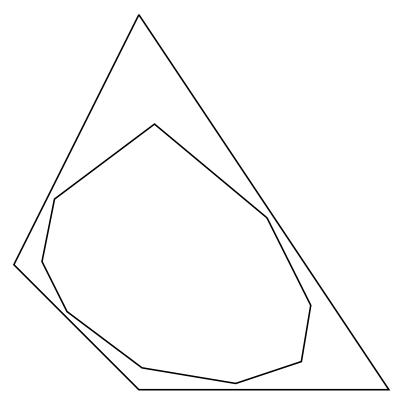

$\operatorname{Area}^{(4)}(\theta)$

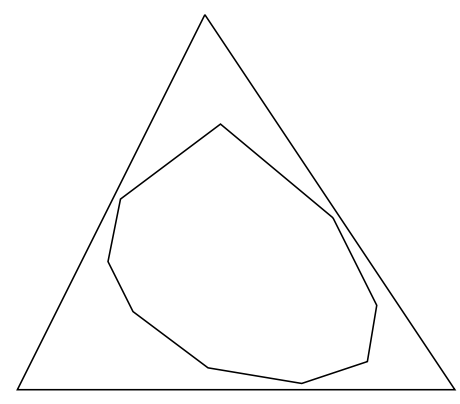

$\operatorname{Area}^{(3)}(\theta)$

Figure 3: Nested areas.

From Lemma 3.1(iii) we know that each of these functions is of the form

$$
\operatorname{Area}^{(i)}(\theta)=a \cdot \cos (2 \theta+b)+c
$$

for constants $a, b$, and $c$ that are independent of $\theta$. Since each function is a cosine function of period $\pi$, each function has exactly two local minima of equal value (and hence they are global minima) over this extended range. Recall that we have reduced proving flushness to showing that any local minima for $\operatorname{Area}^{(m)}(\theta)$ are nonpositive. This will follow from the following two lemmas, which we prove later.

LEMmA 4.1 There exists an angle $\theta^{*}$ such that Area ${ }^{(3)}\left(\theta^{*}\right)=0$. 
LEMMA 4.2 For $3<i \leq m$, and for $0 \leq \theta<2 \pi$, Area ${ }^{(i)}(\theta) \leq$ Area $^{(i-1)}(\theta)$.

The first result says that there is an angle at which Area ${ }^{(3)}$ is nonpositive, and from the second result it follows that all higher order area functions are nonpositive at this angle. Observe that the second lemma is trivial for all angles of interest to the algorithm, but to establish our result we need to show that it is true for all angles in the range 0 to $2 \pi$. Given these two lemmas it follows that the global minimum for $\mathrm{Area}^{(m)}$ must be nonpositive. It only remains to establish the two results listed above. The proofs of both lemmas make use of the following rather remarkable result from classical geometry [8, 10].

Lemma 4.3 (Pivot Lemma) Let $A^{\prime} B^{\prime} C^{\prime}$ be any (nondegenerate) triangle and let $A$, $B$, and $C$ be three points (different from $A^{\prime}, B^{\prime}$, and $C^{\prime}$ ) lying on the extensions of the sides $B^{\prime} C^{\prime}$, $C^{\prime} A^{\prime}$, and $A^{\prime} B^{\prime}$, respectively. Then the three circles $A^{\prime} B C, A B^{\prime} C$, and $A B C^{\prime}$ meet in a unique point $P$, called the Miquel point. (See Fig. 4(a).)

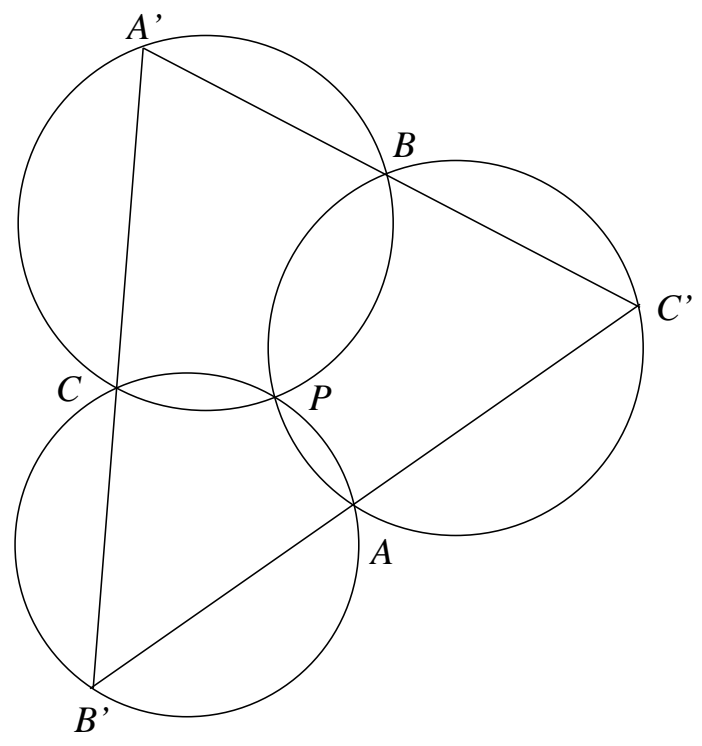

(a)

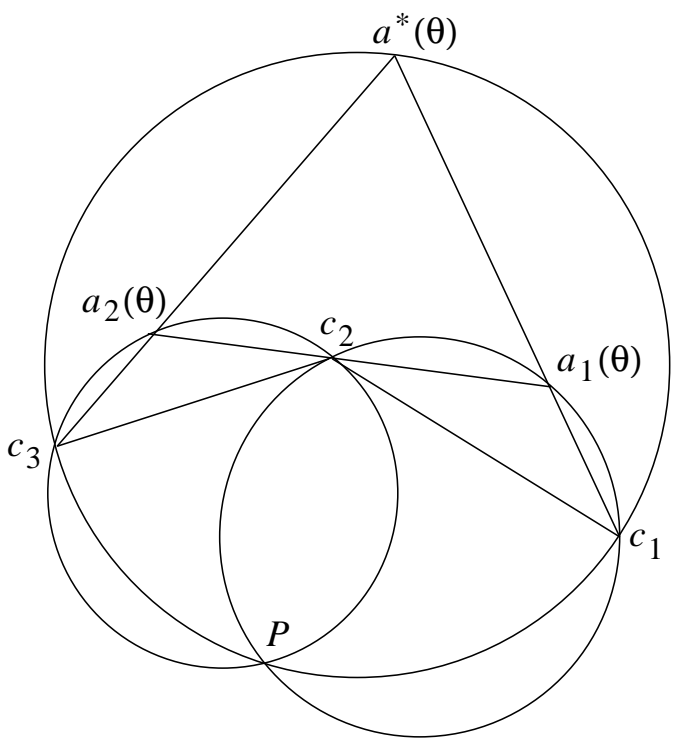

(b)

Figure 4: Pivot Lemma.

Using this lemma we can establish the following result, which describes the behavior of the area function defined by three rotating lines about three contact points. 
Lemma 4.4 Let $A, B$, and $C$ be three noncollinear points in the plane and let $\beta_{a}, \beta_{b}$, and $\beta_{c}$ be three pairwise distinct angles in the range 0 to $\pi$. There is a unique angle $\theta^{*}$ in this same range such that the three lines passing through $A, B$ and $C$ at the angles $\beta_{a}+\theta^{*}, \beta_{b}+\theta^{*}$ and $\beta_{c}+\theta^{*}$, respectively, pass through a common point.

Proof: For $0 \leq \theta<\pi$, let $A^{\prime}(\theta)$ denote the intersection point of the lines passing through $B$ and $C$ at the angles $\beta_{b}+\theta$ and $\beta_{c}+\theta$, respectively. Define $B^{\prime}(\theta)$, and $C^{\prime}(\theta)$ analogously. Observe that because of the angle constraint between pairs of lines, as $\theta$ varies from 0 to $\pi, A^{\prime}(\theta)$ travels continuously, counterclockwise along a circle passing through $B$ and $C$. Analogous statements hold for $B^{\prime}(\theta)$ and $C^{\prime}(\theta)$. Considering any fixed $\theta$, and applying the Pivot Lemma, we see that these circles pass through the Miquel point, $P$.

We argue that all three intersection points arrive at the Miquel point for the same value of $\theta$. Consider the angle $\theta^{*}$ at which $A^{\prime}\left(\theta^{*}\right)$ reaches $P$. For this angle the lines passing through $B$ and $C$ intersect at $P$. Consider the point $C^{\prime}\left(\theta^{*}\right)$. We know that this point lies on the circle $A B C^{\prime}$, and it lies on the line passing through $B$ (which also passes through $P)$. Hence $C^{\prime}(\theta)$ lies on the intersection of the lines $B P$ and the circle $A B C^{\prime}$, that is, either $P$ or $B$. Applying a similar argument to $B^{\prime}\left(\theta^{*}\right)$ shows that it is equal to either $P$ or $C$. If $C^{\prime}\left(\theta^{*}\right)=P$ or $B^{\prime}(\theta)=P$ then we are done, because either implies that all three lines intersect at $P$. Otherwise $C^{\prime}\left(\theta^{*}\right)=B$ and $B^{\prime}\left(\theta^{*}\right)=C$. However because these two points lie on the line passing through $A$, this would imply that $A, B$ and $C$ are collinear, contradicting our initial hypothesis.

This shows the existence of $\theta^{*}$. To prove the uniqueness of $\theta^{*}$ over the range 0 to $\pi$, observe that the functions $A^{\prime}(\theta), B^{\prime}(\theta)$ and $C^{\prime}(\theta)$ all have a period of $\pi$, and over this period they intersect the only possible point of coincidence, $P$, exactly once.

Proof (of Lemma 4.1.): Let $c_{1}, c_{2}$ and $c_{3}$ be the contact points and $a_{1}(\theta), a_{2}(\theta)$, and $a_{3}(\theta)$ be the vertices of the extended envelope, so that $a_{i}(\theta)$ lies between $c_{i}$ and $c_{i+1}$ (indices taken cyclically). As we observed before, as $\theta$ varies from 0 to $\pi, a_{i}(\theta)$ traces out a circle passing through $c_{i}$ and $c_{i+1}$. Applying Lemma 4.4 to the extended envelope for any fixed $\theta_{0}$ (where $A B C$ are the contact points), we find that there is a unique angle $\theta^{*}$ at which the vertices 
$a_{i}\left(\theta^{*}\right)$ intersect in a common point. At this angle we have Area ${ }^{(3)}\left(\theta^{*}\right)=0$.

Proof (of Lemma 4.2.): Suppose that the difference between the extended envelopes defining Area $^{(i)}(\theta)$ and Area ${ }^{(i-1)}(\theta)$ is the removal of contact point $c_{1}$ and its incident side. Let $a^{*}(\theta)$ denote the intersection of the extensions of the edges $c_{1} a_{1}(\theta)$ and $c_{3} a_{2}(\theta)$. (See Fig. 4(b).) Observe that the difference between $\operatorname{Area}^{(i)}(\theta)$ and $\operatorname{Area}^{(i-1)}(\theta)$ is the area of triangle $a_{1}(\theta) a^{*}(\theta) a_{2}(\theta)$. We show that this triangle is of positive (signed) area for all $\theta$ in the range 0 to $\pi$. To see this, observe that the area is positive for some $\theta$ (in particular any $\theta$ in the interval between two critical angles). Because the area of the triangle varies continuously with $\theta$ with a period of $\pi$, if the area becomes negative, there must be at least two angles in this period at which the area is equal to zero. Since the triangle is bounded by three lines which pass through noncollinear points, $c_{1}, c_{2}$ and $c_{3}$, it follows that the area can equal zero only if all its vertices coincide at a common point. However, by applying Lemma 4.4 to the contact points it follows that there is a only one angle over this range at which the vertices coincide, and thus the area is never negative.

\section{Conclusions}

We have presented an $O(n m \log m)$ algorithm for computing the smallest area $m$-envelope enclosing a convex polygon with $n$ sides, whose sides obey a given sequence of exterior angle constraints. The algorithm is a simple application of the method of rotating calipers. The principal subproblems are those of maintaining a data structure that allows the area to be

updated efficiently (which we showed can be done in $O(\log m)$ time per event), and proving the flushness criterion, which states that the minimum area is achieved when at least one edge of the envelope is collinear with an edge of the polygon.

One open problem suggested by this work is how to solve the problem with weaker angle constraints. For example, computing the smallest enclosing parallelogram can be stated in terms of angle constraints which force opposite edges to be parallel, but the angles between adjacent edges are not specified. Can this problem be solved efficiently if edge orientations 
are specified by a system of linear equations, for example? Another important direction for future research involves computing minimum enclosures in three dimensions with given dihedral angles. For example, O’Rourke has given a finiteness characterization of enclosing rectangular boxes [13], but it is unknown whether this criterion can be applied to shapes with arbitrary angle constraints.

\section{References}

[1] A. Aggarwal and J. Park. Notes on searching in multidimensional monotone arrays. In Proc. 29th Annu. IEEE Sympos. Found. Comput. Sci., pages 497-512, 1988.

[2] J. E. Boyce, D. P. Dobkin, R. L. Drysdale, III, and L. J. Guibas. Finding extremal polygons. In Proc. 14th Annu. ACM Sympos. Theory Comput., pages 282-289, 1982.

[3] S. Chandran and D. M. Mount. A parallel algorithm for enclosed and enclosing triangles. Internat. J. Computational Geometry and Applications, 2:191-214, 1992.

[4] T. H. Cormen, C. E. Leiserson, and R. L. Rivest. Introduction to Algorithms. MIT Press, Cambridge, MA, 1990.

[5] N. A. A. DePano. Polygon approximation with optimzed polygonal enclosures: Applications and algorithms. Ph.D. Thesis, Johns Hopkins Univ., Baltimore, MD, 1985.

[6] N. A. A. DePano and A. Aggarwal. Finding restricted $k$-envelopes for convex polygons. In Proc. 22nd Allerton Conf. Commun. Control Comput., pages 81-90, 1984.

[7] D. P. Dobkin and L. Snyder. On a general method for maximizing and minimizing among certain geometric problems. In Proc. 20th Annu. IEEE Sympos. Found. Comput. Sci., pages $9-17,1979$.

[8] H. G. Forder. Geometry. Hutchinson's University Library, London, 1950.

[9] V. Klee and M. C. Laskowski. Finding the smallest triangles containing a given convex polygon. J. Algorithms, 6:359-375, 1985.

[10] Miquel. Théorèmes de géométrie. Liouville's Journal, 3, 1838. 
[11] D. M. Mount. The densest double-lattice packing of a convex polygon. In J. E. Goodman, R. Pollack, and W. Steiger, editors, Discrete and Computational Geometry: Papers from the DIMACS Special Year, pages 245-262. AMS, Providence, RI, 1991.

[12] D. M. Mount and R. Silverman. Packing and covering the plane with translates of a convex polygon. J. Algorithms, 11:564-580, 1990.

[13] J. O’Rourke. Finding minimal enclosing boxes. Internat. J. Comput. Inform. Sci., $14: 183-199,1985$.

[14] J. O'Rourke, A. Aggarwal, S. Maddila, and M. Baldwin. An optimal algorithm for finding minimal enclosing triangles. J. Algorithms, 7:258-269, 1986.

[15] F. P. Preparata and M. I. Shamos. Computational Geometry: An Introduction. SpringerVerlag, New York, 1985.

[16] G. T. Toussaint. Solving geometric problems with the "rotating calipers". In Proc. IEEE MELECON'83, Athens, Greece, 1983. 